\title{
Selective oxidative protection leads to tissue topological changes orchestrated by macrophage during ulcerative colitis
}

\section{Juan Du}

First Affiliated Hospital of Zhejiang University School of Medicine Junlei Zhang

First Affiliated Hospital of Zhejiang University School of Medicine

\section{Xiaocang Cao}

Tianjin Medical University General Hospital, Tianjin Medical University

\section{Xun Wang}

First Affiliated Hospital of Zhejiang University School of Medicine

\section{Jinyuan Song}

First Affiliated Hospital of Zhejiang University School of Medicine

\section{Lin Wang}

Jie Zhang

Department of Gastroenterology, the First Affiliated Hospital, College of Medicine, Zhejiang University Jianghui Tang

First Affiliated Hospital of Zhejiang University School of Medicine

\section{Jiangchao Wu}

First Affiliated Hospital Zhejiang University

\section{Yongtao Ji}

First Affiliated Hospital of Zhejiang University School of Medicine

\section{Yaxing Zhao}

First Affiliated Hospital of Zhejiang University School of Medicine

\section{Fei Cheng}

The First Affiliated Hospital, School of Medicine, Zhejiang University

Jun Li

First Affiliated Hospital of Zhejiang University School of Medicine

Irene Soncin

Nanyang Technological University

\section{Vincent Tano}

Lee Kong Chian School of Medicine, Nanyang Technological University

\section{Guang Li}

Beijing Chaoyang Hospital, Capital Medical University, Chaoyang District 


\section{Xinjuan Liu}

Beijing Chaoyang Hospital, Capital Medical University, Chaoyang District

Jianpeng Sheng ( $\nabla$ shengjp@zju.edu.cn )

First Affiliated Hospital of Zhejiang University School of Medicine

\section{Article}

Keywords: macrophage, single-cell sequencing, imaging mass cytometry

Posted Date: August 13th, 2021

DOl: https://doi.org/10.21203/rs.3.rs-763356/v1

License: (c) (i) This work is licensed under a Creative Commons Attribution 4.0 International License. Read Full License 


\section{Abstract}

Background and Aims: Ulcerative colitis(UC) is a chronic inflammatory bowel disorder with highly cellular heterogeneity. Mass cytometry(Cy-TOF) and single-cell RNA sequencing (scRNA-seq) have revealed cellular heterogeneity of UC. However, comprehensive elucidation of tissue topological changes within the UC ecosystem is still missing. And we aimed to illustrate compositional and spatial changes of the UC ecosystem.

Methods: Imaging mass cytometry (IMC) and scRNA-seq were applied to depict the single-cell landscape of colon ecosystem.

Results: We noticed tissue topological changes featured with macrophage disappearance reaction (MDR) in UC region. MDR only occurred for $\mathrm{CD}_{163^{+}}$tissue-resident macrophages. We found reactive oxygen species (ROS) level were higher in UC region but ROS scavenging enzyme SOD1/2 were barely detected in resident macrophages, resulting selective oxidative protection for inflammatory macrophages and resident macrophage disappearance reaction. Furthermore, inflammatory macrophages replaced resident macrophages during UC, which played a key role in forming the inflammatory cellular network by producing TNF- $\alpha$ and IL-1 $\beta$.

Conclusions: Our study dissected the microenvironment of UC lesions at single-cell resolution while preserving its architecture, based on which, we discovered the mechanism of MDR in UC region and resident macrophage specific MDR resulted in infiltration of inflammatory macrophage, which formed the cytokine producing network within the local cellular neighborhood.

\section{Introduction}

Ulcerative colitis (UC) is a chronic idiopathic inflammatory bowel disorder of the colon featuring continuous mucosal inflammation, affecting millions of people worldwide, and its incidence has increased over recent years ${ }^{1}$. Tissue damage in UC patients is mainly driven by a dysregulated immune response. Various types of immune cells, such as monocytes, macrophages, and T cells, play a crucial role in UC pathogenesis ${ }^{2}$.

Macrophage in the lesion is important for UC development and treatment. We and others have shown that macrophages in the intestinal space consist of resident and infiltrating inhabitants ${ }^{3}$, and resident macrophages are believed to suppress the inflammation during UC ${ }^{4}$ while infiltrating monocytes give rise to proinflammatory effector cells 5 .

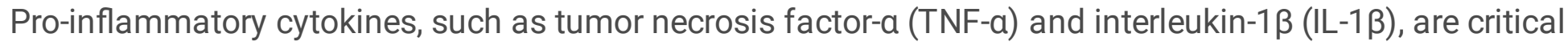
for disease progression as well ${ }^{6}$. And TNF-a neutralizing antibody treatment for $\mathrm{UC}$ has become favorable in the past decade ${ }^{7}$ due to its successful application in the induction and maintenance of UC remission. Macrophage was reported to be involved in the TNF-a mediated UC ${ }^{8,9}$. However, many 
patients are still resistant to anti-TNF-a therapy ${ }^{10}$. Thus, comprehensive elucidation of macrophage population change during UC is important for understanding and intervention of UC.

The macrophage disappearance reaction (MDR) was first noticed in response to certain stimuli such as inflammation and infection ${ }^{11}$, but its mechanism was only illustrated partially in a recent report ${ }^{12}$ that peritoneal macrophage disappeared after bacterial infection based on factor $\mathrm{V}$ dependent coagulation process. However, if MDR happens in UC and whether same coagulation process occurred during UC progression was yet not understood.

High-dimensional single-cell analytics such as mass cytometry (Сy-TOF) and single-cell RNA sequencing (scRNA-seq) have revealed cellular heterogeneity in UC $13,14,15$ and have identified many important cellular subsets promoting or attenuating $\mathrm{UC}$, such as $\mathrm{CD} 14^{+}$macrophage ${ }^{16}, \mathrm{IL}-17^{+} \mathrm{CD} 8^{+} \mathrm{T}$ cells ${ }^{13}$, and $\mathrm{CD} 26^{+} \mathrm{CD} 8^{+} \mathrm{T}$ cells ${ }^{17}$. However, the tissue topology, such as spatial distribution and neighborhood relations of different cells, is lost during both Cy-TOF and scRNA-seq analysis, which rely on dissociated cells. And the spatial immune network is yet to be defined. In contrast to scRNA-seq and CyTOF analyses, imaging mass cytometry (IMC) enables spatial resolved phenotyping of the UC ecosystem.

Here, we aimed to dissect the tissue UC ecosystem while preserving its architecture so that we might understand how tissue topology changes during UC progression. Using a combination of single-cell transcriptomics and proteomics method, we resolved both cellular and spatial heterogeneity of the UC microenvironment at single-cell resolution. Additionally, we noticed macrophage disappearance reaction (MDR) in UC region, but only occurred for $\mathrm{CD} 163^{+}$tissue-resident macrophages and IL- $1 \beta^{+}$inflammatory macrophage replaced $\mathrm{CD} 163^{+}$tissue-resident macrophage, which played a key role in forming the inflammatory cytokine network of TNF- $a$ and IL-1 $\beta$. We found MDR in UC was not based on factor V dependent coagulation process but due to uneven expression of ROS scavenging enzyme SOD $1 / 2$ resulted oxidative stress. Our study might shed light on the potential macrophage-based immunotherapy for UC.

\section{Materials And Methods}

\section{Ethics statement}

The study followed the Declaration of Helsinki principles and was approved by the Medical Ethics Committee of the First Affiliated Hospital, Zhejiang University, Hangzhou, China. Samples were obtained from all patients by surgical resection or biopsies with informed consent. Animal experiments were approved by the Animal Care and Medical Ethics Committee of the First Affiliated Hospital, Zhejiang University.

\section{Animal Studies}

C57BL/6J mice were purchased from the Model Animal Research Center of Nanjing University (China). All mice were bred in an SPF facility under the regulation of the Institutional Animal Care \& Use Committee 
(IACUC).

\section{DSS-Induced Colitis Model}

Male mice at 4-6 weeks of age were randomly divided into two groups which were oral administration with $1.5 \%$ or $3 \%(\mathrm{w} / \mathrm{v})$ Dextran sulfate sodium (DSS; Sigma, 42867) dissolved in drinking water respectively for one week, followed by normal water for a four-week recovery phase. Changes of bodyweight were monitored every day to identify the successful of mice model throughout the experimental period. After five weeks, all mice were sacrificed and the entire colon were collected for immunohistochemistry analysis and Fluorescence-activated cell sorting (FACS) analysis.

\section{IMC analysis}

Paraffin-embedded and formalin-fixed tissues from UC patients by biopsies were cut into $4 \mu \mathrm{m}$ sections followed by heating at $68^{\circ} \mathrm{C}$ for 1 hour. Dewaxing in xylene was performed twice for 10 min each. Sections were rehydrated sequentially in $95 \%, 85 \%$, and $75 \%$ ethanol for 5 min each, followed by $100^{\circ} \mathrm{C}$ heat-mediated antigen retrieval for $30 \mathrm{~min}$. After cooling naturally, sections were washed twice for $5 \mathrm{~min}$ each with PBS-TB (PBS with 0.5\% Tween-20 [93773, Sigma-Aldrich] and 1\% Bovine Serum Albumin [SRE0098, Sigma-Aldrich]). Then, sections were blocked with SuperBlock (37515, Thermo Fisher Scientific) for $30 \mathrm{~min}$. Following another three PBS-TB washes, sections were incubated with an antibody cocktail at $4^{\circ} \mathrm{C}$ overnight. The antibodies were mostly labeled with metals using the Maxpar ${ }^{\circledR} \mathrm{X} 8$ Antibody Labeling Kit (Fluidigm). Briefly, an X8 polymer was linked to the reduced antibody, and a specific metal was loaded onto the polymer. The final product panel is presented in Supplementary Table S1. After antibody incubation overnight at $4^{\circ} \mathrm{C}$, three additional PBS-TB washes were performed. The sections were incubated with a $1.25 \mu \mathrm{M}$ Intercalator-Ir solution (201192B, Fluidigm) in PBS-TB for $30 \mathrm{~min}$ at RT to label nuclei, followed by two PBS-TB washes and one final $\mathrm{ddH}_{2} \mathrm{O}$ wash. An imaging mass cytometer (Fluidigm, Hyperion) was employed to scan the prepared sections to generate the multiplexed images. For segmenting image data into single-cell data, we used CellProfiler software (Whitehead Institute for Biomedical Research and MIT's CSAIL) to acquire mask files, which were put into histoCAT software for tSNE, PhenoGraph, and cell neighborhood analyses.

\section{Cell neighborhood analysis}

The primary neighbors of itself and secondary neighbors of its primary neighbors was calculated by cell neighborhood analyses as previously reported ${ }^{18}$. Cellular composition was analyzed for each cell

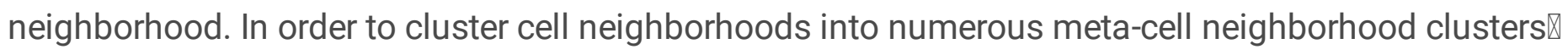
PhenoGraph was then applied to this cellular composition matrix.

\section{Fluorescence-activated cell sorting (FACS) analysis}

Tissue obtained by biopsies or mice colon were digested in culture medium supplemented with 0.6 $\mathrm{mg} / \mathrm{mL}$ collagenase IV (17104019, Gibco) and $0.01 \mathrm{mg} / \mathrm{mL}$ DNase I (11284932001, Merck) in a 170-rpm 
constant temperature shaker (Eppendorf) at $37^{\circ} \mathrm{C}$ for 1 hour. Digested tissues were passed through a 0.4 $\mu \mathrm{m}$ cell strainer $(352340, \mathrm{BD})$ to acquire single-cell suspensions before centrifuging at $300 \mathrm{~g}$ for $5 \mathrm{~min}$ and resuspending in 35\% Percoll (P4937, Sigma). After another centrifugation at $500 \mathrm{~g}$ for $5 \mathrm{~min}$, cells were resuspended in $10 \mathrm{~mL}$ of blood lysis buffer $(555899, \mathrm{BD})$ for $10 \mathrm{~min}$ at RT to eliminate red blood cells. Cell suspensions were centrifuged at $300 \mathrm{~g}$ for $5 \mathrm{~min}$ and then incubated with $2.5 \mu \mathrm{g} / \mathrm{mL}$ Fc blocker (156604 mouse; 422302 human, BioLegend) on ice for $15 \mathrm{~min}$. The suspensions were further incubated with fluorochrome-labeled antibodies at $4^{\circ} \mathrm{C}$ for $30 \mathrm{~min}$. The antibody panel is listed in Supplementary Table S1. The samples were washed and resuspended in PBS and supplemented with $2 \%$ FBS for analysis on a five-laser flow cytometer (BD Bioscience, Fortessa). The data were analyzed with FlowJo software (TreeStar).

\section{Single cell RNAseq analysis}

Single cells were dissociated from the gut samples obtained by biopsies through the method mentioned in the FACS analysis. Briefly, the single cells were loaded into Chromium microfluidic chips with v3 chemistry, then barcoded with a 10x Chromium Controller (10X Genomics, Pleasanton, CA, USA). RNA from the barcoded cells was subsequently reverse-transcribed and sequencing libraries were constructed with reagents from a Chromium Single Cell v3 reagent kit (10X Genomics) according to the manufacturer's instructions. Library sequencing was performed at Novogene Co., Ltd (Tianjin Novogene Technology Co., Tianjin, China) with Illumina HiSeq 2000 according to the manufacturer's instructions (Illumina, San Diego, USA).

\section{Statistical analysis}

Raw data obtained from FACS and histoCAT were copied into GraphPad software. Statistical tests were selected based on the appropriate assumptions for the data distribution and variability characteristics. Sample data were analyzed by a two-tailed Student's $t$-test to identify statistically significant differences between the two groups. One-way ANOVA with the Bonferroni post-test was used to identify differences among three or more groups. The data are represented as the means \pm SEMs. A $p$-value $<0.05$ indicated statistical significance.

\section{Results}

\section{Spatial resolved portrait of UC ecosystem}

In order to dissect the spatial heterogeneity of the UC microenvironment while preserving the architecture of the UC ecosystem, we established a 39-marker IMC panel for UC ecosystem (Figure 1A,B), based on published protocols for intestinal tissue and cancer 19, 20, 21, 22 . Our 39-marker IMC panel covered markers for epithelial, endothelial, stromal cells, and various immune cells; the cytokines IL-1 $\beta$, TNF-a, and IL-6; the proliferation marker Ki-67; and the apoptotic marker cleaved caspase-3 (Supplementary Table S1), which allowed highest level of multiplexing of IMC so far. 
We first analyzed colon tissues of healthy donors, which revealed typical structural markers, such as Collagen I and CD31, immunological markers, such as CD3 and CD20 (Figure 1B), and cytokines, such as TNF- $\alpha$ and IL-1 $\beta$ after staining for the 39-marker panel (Figure 1B).

Distinct histological features, including intestinal crypts revealed by Pan-keratin staining (cyan) of epithelial cells and smooth muscle layer revealed by aSMA staining (yellow) of smooth muscle cells, were consistent with those determined by hematoxylin and eosin (H\&E) staining but were resolved in greater detail (Figure 1C).

We detected major cell types, including $\mathrm{CD}_{3} 1^{+}$endothelial cells, $\mathrm{aSMA}^{+}$smooth muscle cells, and Collagen I deposition in the extracellular matrix (Figure 1C). Immune cell types detected included B cells $\left(\mathrm{CD} 20^{+}\right), \mathrm{T}$ cells $\left(\mathrm{CD} 3^{+}\right)$. Notably, $\mathrm{T}$ cells and $\mathrm{B}$ cells were highly enriched in the intraepithelial space and gland surrounding area (Figure 1D). Resident macrophages (CD163 $\left.{ }^{+} \mathrm{CD}_{6} 8^{+} \mathrm{CD} 16^{+} \mathrm{CD} 11 \mathrm{~b}^{\text {low }}\right)$, and inflammatory macrophages $\left(\mathrm{CD} 163^{-} \mathrm{CD} 8^{+} \mathrm{CD} 16^{-} \mathrm{CD} 11 \mathrm{~b}^{+}\right){ }^{22}$ could be detected mainly underneath the epithelial cells (Figure 1E).

Furthermore, to characterize the cellular heterogeneity of the UC microenvironment while preserving gross structural features, formalin-fixed, paraffin-embedded (FFPE) human tissue samples from 9 healthy donors and 23 UC patients with different Mayo grading scores (Supplementary Table S2) were processed and stained with 39 metal-conjugated antibodies, before scanning by IMC. The scanned images were subjected to cell segmentation using the established bioinformatic tool, histoCAT, developed by Schapiro et al. ${ }^{23}$ (Figure 1A). The expression of markers in each cell was quantified, and 26 cell clusters were identified by PhenoGraph (Figure 1F), which were then annotated based on the expression of key markers.

CD45- Pan-keratin ${ }^{+}$cells indicated epithelial cells (Supplementary Figure S1A), endothelial cells were defined by CD31 and Vimentin staining (Supplementary Figure S1B), smooth muscle cells were identified by aSMA ${ }^{+}$cells (Supplementary Figure S1C), and $C D 68^{+} C D 163^{+} C D 11 b^{\text {low }}$ and $C D 68^{+} C D 163^{-} C D 11 b^{+}$cells were defined as resident and inflammatory macrophages, respectively (Supplementary Figure S1D), which was consistent with scRNA data (Supplementary Figure S2). T cells and B cells were concentrated in the intraepithelial space (Figure 1D), thus, T cell, B cell, and epithelial markers overlapped due to the resolution limit of IMC (Supplementary Figure S1E). However, T cells and B cells can be separated from epithelial cells based on CD45 expression (Supplementary Figure S1E). Overall, the T cell, B cell, and NK cell markers showed co-staining in clusters $6,7,9,18$, and 22, which were defined as lymphocytes (Figure $1 \mathrm{~F})$. FoxP $3^{+} \mathrm{CD} 25^{+}$cells were defined as regulatory $\mathrm{T}$ cells (Supplementary Figure S1F). In total, the 26 cell clusters included 7 from epithelial cells, 6 from endothelial cells, 1 from smooth muscle cells, 11 from immune cells, and 1 from unstained cells. Their frequencies in different patients are shown in Figure 1G.

In summary, tissue architecture, major stromal cell types, diverse immune cell populations, and cytokine production in the human colon microenvironment could be observed with our 39-marker panel by IMC 
and our IMC results revealed a detailed landscape of UC ecosystem, including epithelial cells, stromal cells, and various immune cells.

\section{MDR occurs only for resident macrophage during UC progression}

Next, we wanted to characterize cellular changes during UC progression. Firstly, 9 healthy donors and 23 UC patients' samples were separated into 3 major classes based on their cellular frequencies (Figure $2 A, B)$. All samples from the 9 healthy donors were grouped together (green) whereas, samples from UC patients were separated into mild (blue, Mayo grade 1) and severe groups (red, Mayo grade 2-4), Mayo grade was determined by the patients' colonoscopy (Figure 2C).

Further analysis showed that epithelial cells decreased from healthy donors to severe UC patients (Figure 2D; clusters 4 and 23), perhaps due to increased tissue damage, which was also confirmed by flow cytometry analysis of colon biospecimen (Figure 2E). While lymphocytes increased (Figure 2F; clusters 6, 18 , and 22), indicating increased lymphocyte infiltration due to inflammation. Since the resolution of IMC for lymphocytes in UC region was poor, we also performed flow cytometry analysis for UC samples, and we noticed heavy infiltration of T cells and B cells, especially for B cell and CD4 ${ }^{+} T$ cells (Figure $2 G$ ).

Furthermore, we also noticed that the macrophage disappearance reaction occurred only for resident macrophage during UC progression (Figure $2 \mathrm{H}$; cluster 11, 14, and 24). While inflammatory macrophages (Figure 2J; cluster 25) increased when UC developed, suggesting a population switch occurred in the macrophage system. Macrophage disappearance reaction (MDR) was confirmed in UC patients' samples by FACS. $C D 68^{+} \mathrm{CD} 11 \mathrm{~b}^{-}$resident macrophages almost disappeared in UC patients while $\mathrm{CD} 68^{+} \mathrm{CD} 11 \mathrm{~b}^{+} \mathrm{CCR} 2^{+} \mathrm{HLA}-\mathrm{DR}{ }^{+}$inflammatory macrophages increased significantly (Figure $2 \mathrm{~K}$ ).

Both IMC and FACS data revealed a change in cellular components with UC progression, especially for the macrophage system, where inflammatory macrophages replaced resident macrophages.

\section{Macrophage replacement kinetics during UC}

To analyze the macrophage replacement kinetics during UC, we used the canonical mouse DSS model (Figure $3 \mathrm{~A})^{4,17,24}$. We found that $\mathrm{CD} 11 \mathrm{~b}^{+} \mathrm{F} 4 / 80^{\text {hi }}$ Fraction I cells represented colon tissue-resident

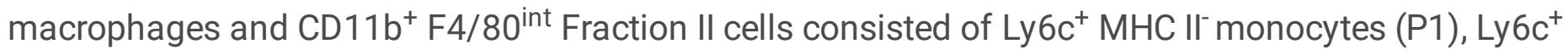
$\mathrm{MHC} \mathrm{II}^{+}$inflammatory macrophages (P2), Ly6c $\mathrm{C}^{-} \mathrm{MHC} \mathrm{II}^{+}$infiltrating macrophages (P3), and Ly $6 \mathrm{c}^{-} \mathrm{MHC}$ $\mathrm{II}^{-}$eosinophils (P4), as described previously ${ }^{3}$ (Figure 3B).

Additionally, we performed a time point analysis for resident and inflammatory macrophages in an acute DSS model. 1.5\% and 3\% DSS treatments represented mild and severe UC (Figure 3C). Similar macrophage replacement kinetics were observed for both the mild and severe DSS models, where 
resident macrophages reduced to a minimum level around day 9 before returning to a normal level around day 28 (Figures $3 \mathrm{C}, \mathrm{D}$, and E). Simultaneously, $\mathrm{Ly}_{6 \mathrm{C}^{+}} \mathrm{MHC} \mathrm{I}{ }^{+}$inflammatory macrophages reached a maximum around day 9 and returned to a basal level around day 14, while Ly $6 \mathrm{c}^{+} \mathrm{MHC} \mathrm{II}^{-}$monocytes reached the basal level slightly later at around day 21 (Figure 3C, D, and E). Ly $6 \mathrm{c}^{-} \mathrm{MHC} \mathrm{I}{ }^{+}$infiltrating macrophages and $\mathrm{CD} 11 \mathrm{~b}^{+} \mathrm{F} 4 / 80^{-}$neutrophils showed different recruitment kinetics during mild and severe UC. Ly6c ${ }^{-} \mathrm{MHC} \mathrm{II}^{+}$infiltrating macrophages decreased to the minimum level around day 9 , similar to $\mathrm{CD} 11 \mathrm{~b}^{+} \mathrm{F} 4 / 80^{\text {hi }}$ resident macrophages, and replenishment of $\mathrm{Ly} 6 \mathrm{c}^{-} \mathrm{MHC} \mathrm{II}^{+}$infiltrating macrophages was completed around day 35 for mild UC but took longer for severe UC (Figures 3C, D, and E). Neutrophil recruitment reached a maximum between days 7 to 14 , which was only resolved around day 28 for mild UC and much later for severe UC (Figures 3C, D, and E). It is well known that monocyte recruitment is dependent on the chemokine CCR2, thus we wanted to try if we could disrupt the inflammation network formation through CCR2 knockout. However, the Ly $6 \mathrm{c}^{+} \mathrm{MHC} \mathrm{II}{ }^{+}$macrophage infiltration into the inflammatory region was not affected (Figure 3F), which was probably due to complementary roles of other chemokines like CCR5.

Overall, our dynamic analysis of macrophage replacement using the mouse DSS model showed that inflammatory macrophages could replace resident macrophages during DSS treatment and return to a basal level when inflammation was resolved. In addition, CCR2 knockout was not enough to block the mouse inflammatory macrophage infiltration.

\section{scRNA-seq analysis confirms MDR of the UC ecosystem}

To confirm the IMC pathological landscape of the UC microenvironment and illustrate the mechanism of MDR, scRNA-seq analysis was performed on four healthy donors' colon, four UC patients' self-control and corresponding lesion samples (Figure 4A). 52716 single cells were captured with high sequencing quality and we found 15 major cell clusters (Figure. 4B), which was annotated based on their specific markers (Figure 4C), such as $\mathrm{EpCAM}^{+}$epithelial cells (Supplementary Figure. S2), $\mathrm{DCN}^{+}$smooth muscle cells ${ }^{25}$, $\mathrm{CD} 31^{+}$endothelial cells and rare Gnat $3^{+}$Tuft cell (Supplementary Figure S2A) ${ }^{26}$. In addition, various immune cell subsets were identified, including $\mathrm{CD} 4^{+}, \mathrm{CD}^{+} \mathrm{T}$ cells, $\mathrm{FoxP}^{+}$regulatory $\mathrm{T}$ cells (Treg) and IL7R ${ }^{+}$memory T cells (Figure. 4B and Supplementary Figure S2B). B cells could also be separated into IgG producing plasma cells, $\mathrm{CD} 83^{+}$activated $\mathrm{B}$ cells ${ }^{27}$ and Lymphotoxin $\beta^{+} \mathrm{B}$ cells (Supplementary Figure S2B) ${ }^{28}$. Furthermore, macrophages were classified into $C D 68^{+} C D 163^{+} \mathrm{CD} 11 \mathrm{~b}^{\text {low }}$ resident macrophage and $\mathrm{CD} 68^{+} \mathrm{CD} 11 \mathrm{~b}^{+} \mathrm{IL}-1 \beta^{+}$inflammatory macrophages. $\mathrm{CD} 11 \mathrm{~b}^{+}$inflammatory macrophage expressed higher level of CCR2, which was central chemokine for macrophage recruitment (Supplementary Figure S2B).

We also observed decreased epithelial cells with heavy infiltration of T cells and B cells, including T cells, $\mathrm{CD} 83^{+}$activated $\mathrm{B}$ cells and plasma cells, in the UC region compared to the control region (Figure 4D). 
And Treg cells were also elevated in UC region (Figure 4D), indicating a negative feedback for the inflammation process in the UC region.

In addition, consistent with the results showed in Figure 2J, the scRNA-seq analysis showed that resident macrophage decreased, while inflammatory macrophages increased in UC region (Figure 4D), indicating the MDR occurred only in the resident macrophage subset ${ }^{11}$. Overall, our scRNA-seq landscape covers the major ICC TME cellular components and depicts the dynamic changes of the intestinal ecosystem, such as the MDR occurred in the UC region, which was specific to resident macrophage, confirming the IMC results.

\section{Uneven expression of ROS scavenging enzyme SOD1/2 resulted ROS induced resident macrophage apoptosis}

Next, we tried to illustrate the mechanism for resident macrophage disappearance during UC progression. First, we tested if MDR observed in UC region also involved the Factor V dependent coagulation process, as previously reported ${ }^{12}$. However, Factor $\mathrm{V}$ was not expressed by resident macrophages or other cells (Figure 5A). In order to explore the mechanism by which resulted in MDR, we analyzed the differentially expressed gene (DEG) between resident macrophage and infiltrating macrophage due to the opposite cellular dynamics between these two macrophage populations observed during UC. Among the DEG『we found differentially expressed of SOD2 which participate in the process of oxidative stress (Figure 5B). Furthermore, we found ROS scavenging enzyme SOD1/2 expression was uneven. SOD1 was generally expressed by many cells except resident macrophage and SOD2 was highly expressed specifically only in inflammatory macrophages (Figure $5 \mathrm{C}$ ). As a result, ROS level was quite high in resident macrophage compared with inflammatory macrophage, detected by FACS compatible ROS probes (Figure 5D).

In UC region, epithelium barrier is usually disrupted and commensal bacterial invades the intestinal space, which results bacterial infection defense system including ROS generation ${ }^{29}$. To mimic this scenario, THP-1 cells were first induced to MO cell with PMA ${ }^{30}$ and then cocultured with bacterial component LPS. LPS induced ROS generation (Figure 5E), as previously reported ${ }^{31}$. Enhanced activation of capsase-3 was observed, upon LPS exposure (Figure 5F), which resulted macrophage apoptosis (Figure 5G), consistent with an earlier report on macrophage ${ }^{32}$.

In general, we found general elevated oxidative phosphorylation and ROS level in the UC region compared with healthy control and self-control. Meanwhile, ROS scavenging enzyme SOD 1/2 were highly expressed only in inflammatory macrophage but not resident macrophage, which resulted ROS induced resident macrophage apoptosis as previously reported ${ }^{33}$.

\section{Cellular neighborhood changes during UC}


It is widely accepted that the changes of macrophages can further influence the nearby immune system in situ ${ }^{34}$. Hence, it is critical to identify the local changes of the inflammation network following the switch of macrophages. Therefore, we performed the regional cellular neighborhood analysis. Cell neighborhood was identified for each cell, primary neighbors within $4 \mu \mathrm{m}$ of the target cells and the secondary neighbors of the primary neighbors were defined as interaction partners of the target population (Figure 6A) ${ }^{19,22}$. In order to figure out different cellular neighborhood function units, cellular neighborhoods were annotated according to the major cellular clusters (Figure 6B). Then based on the CN composition, Voronoi plot was used for each IMC image to visualize whether the defined CN function units were associated with the inflammation caused by UC (Fig 6C) as previously reported ${ }^{35}$. As shown in figure $6 \mathrm{C}$, the topology map of IMC represented by Voronoi plot with various $\mathrm{CN}$ indicated by different colour was aligned nicely with IMC image. We found lymphocyte-enriched $\mathrm{CN}$ and infiltrating macrophage centered $\mathrm{CN}$ were highest in severe UC patients. Besides, we found epithelial-enriched $\mathrm{CN}$ and resident MF-enriched CN were lowest in severe UC patients (Figure 6D).

Thus, CN changes during UC was consistent with the cellular cluster changes.

\section{Infiltrating macrophages recruitment forms the inflammatory cytokine network within the cellular neighborhood}

Next, we intend to explore to the mechanism and results of the $\mathrm{CN}$ changes. We noticed profound cytokine production in UC patients based on both IMC and sCRNA-seq results. IMC showed that TNF-a was produced mainly by lymphocytes (Figure 7A), and scRNA-seq helped to identify the T cells, CD83 ${ }^{+}$ activated B cells and inflammatory macrophages as major TNF-a producers (Figure 7A). In addition, our IMC and scRNA-seq data showed that IL- $1 \beta$ was produced mainly by infiltrating macrophages (Figure 7B).

Furthermore, cell-cell interaction analysis using scRNA-seq data was implemented based on the published tool, NicheNET, by Browaeys et al ${ }^{36}$, which models intercellular communication by linking ligands to target genes. Inflammatory macrophages were shown to induce $T$ cells and B cells to produce TNF- $a$ through IL-1 $\beta$, based on NicheNET analysis (Figure 7C).

Consistent with the scRNA-seq analysis, neighbors of resident macrophage expressed less TNF-a, while neighbors of inflammatory macrophages expressed more TNF-a, As shown in figure 7D, to prove the crucial role of the infiltrating macrophage in formation of inflammation network, we sorted the $\mathrm{Ly}^{6} \mathrm{c}^{+} \mathrm{MHC}$ $\mathrm{II}^{+}$macrophage from DSS treated mouse and co-cultured with the lymphocytes from the draining mesenteric lymph nodes (Figure 7E). And we found co-culture with $\mathrm{Ly}_{6 \mathrm{C}}{ }^{+} \mathrm{MHC} \mathrm{II}$ macrophage could induce both T cells and B cells to secrete TNF-a (Figure 7E).

In summary, by combining IMC and scRNA-seq data, we suggested that during UC, bacteria invasion caused ROS production led to resident macrophage specific MDR and infiltration of inflammatory 
macrophage, which formed the inflammatory $\mathrm{CN}$ with lymphocytes and orchestrated the inflammatory cytokine network (Figure 7F).

\section{Discussion}

The UC microenvironment is composed of epithelial cells, immune cells, and stromal cells. Recently, scRNA-seq has helped resolve the heterogeneity of the UC microenvironment among different patients at the transcriptional level ${ }^{16,17,37}$. However, single-cell spatial heterogeneity of the UC microenvironment remains to be deciphered. Here, we combined ScRNA-seq analysis and IMC with 39 protein markers to analyze UC heterogeneity in great detail, while preserving the architecture of the microenvironment. We generated the first single-cell pathology landscape collection of human UC. Furthermore, we discovered that MDR occurred only for resident macrophages and inflammatory macrophages the replaced resident macrophages, forming the cytokine network of TNF- $\alpha$ and IL- $1 \beta$ with T cells and $C D 83^{+} B$ cells. And resident macrophage apoptosis was due to selective oxidative stress exposure.

We showed that the MDR occurred in both human and mouse UC for $C D 163^{+}$and $C D 11 b^{+} F 4 / 80^{\text {hi }}$ resident macrophages, respectively. In addition, resident macrophages only returned to normal levels when inflammation was resolved in the mouse DSS model. Thus, chronic inflammation might prevent the replenishment of resident macrophages, the major immune suppressive cells, in UC lesions of patients. We also noticed that IL-1 $\beta^{+}$inflammatory macrophages predominated over resident macrophages in the DSS mouse model and formed the inflammatory cytokine network with T cells and B cells. Our findings were consistent with a previous report, which showed that CD14 ${ }^{+}$macrophages in Crohn's disease consisted of IL-1 $\beta$ producing CD $163^{-/ \mathrm{dim}}$ cells ${ }^{16}$. We believed such macrophage replacement dynamics was originally adopted as the combinatory bacterial defense mechanism that ROS was elevated as the first defense of the invading bacteria. At the same time, immune suppressive resident macrophage was wipe out purposely due to their sensitivity to ROS and replaced by inflammatory macrophage which was resistant to ROS based on high level of SOD1/2. However, if the inflammation process was not resolved properly, persisted loss of resident macrophage and infiltration of inflammatory macrophage would exacerbate UC.

The resolution of IMC was limited for the regions with high density of cells. However, single-cell proteomics and transcriptomics analysis did complement each other. For example, when T cell and B cell markers were overlapped in areas of high cell density, scRNA-seq could resolve T cells and B cells effectively. Also, scRNA-seq was unable to determine the location of cytokine production while IMC revealed that TNF-a producing lymphocytes were surrounding the inflammatory macrophage but not resident macrophages.

In summary, we not only provide novel spatial insights into the ecosystem but also identify oxidative stress as the key player responsible for the UC macrophage subset balance during UC. And future immunomodulation therapeutic application could be developed to modulate the $\mathrm{CN}$ based on our tissue topological analysis presented here. 


\section{Declarations}

\section{Funding}

This work was supported by the National Key Research and Development Program of China (grant 2019 YFA0803000 to J.S.), the Zhejiang Provincial Natural Science Foundation (Y19H030059 to J.D.), and the National Natural Science Foundation of China (grant 82000489 to J.D., grant 82070559 to X.L.).

\section{Acknowledgements}

We are grateful to the participating patients for their contribution to our research. And we also thank the collaborators in Institute of Pharmaceutics, College of Pharmaceutical Sciences of Zhejiang university. The authors would also like to thank OE Biotech Co, Ltd (Shanghai, China) for the technical support for the single cell analysis.

\section{Declaration of interests}

The authors declare no competing interests.

\section{Data availability statement}

The data underlying this article are available in BioProject titled as Ulcerative colitis(UC) scRNA-seq, and can be accessed with PRJCA005436/CRP002957.

\section{References}

1. Vegh Z, Kurti Z, Lakatos PL. Epidemiology of inflammatory bowel diseases from west to east. $J$ Dig Dis 18, $92-98$ (2017).

2. Gren ST, Grip O. Role of Monocytes and Intestinal Macrophages in Crohn's Disease and Ulcerative Colitis. Inflamm Bowel Dis 22, 1992-1998 (2016).

3. Sheng J, Ruedl C, Karjalainen K. Most Tissue-Resident Macrophages Except Microglia Are Derived from Fetal Hematopoietic Stem Cells. Immunity 43, 382-393 (2015).

4. Zigmond E, et al. Macrophage-restricted interleukin-10 receptor deficiency, but not IL-10 deficiency, causes severe spontaneous colitis. Immunity 40, 720-733 (2014). 
5. Zigmond $\mathrm{E}$, et al. Ly6C hi monocytes in the inflamed colon give rise to proinflammatory effector cells and migratory antigen-presenting cells. Immunity 37, 1076-1090 (2012).

6. Park JH, Peyrin-Biroulet L, Eisenhut M, Shin JI. IBD immunopathogenesis: A comprehensive review of inflammatory molecules. Autoimmun Rev 16, 416-426 (2017).

7. Kunovszki $\mathrm{P}$, et al. Epidemiological data and utilization patterns of anti-TNF alpha therapy in the Hungarian ulcerative colitis population between 2012-2016. Expert Opin Biol Ther 20, 443-449 (2020).

8. Lissner D, et al. Monocyte and M1 Macrophage-induced Barrier Defect Contributes to Chronic Intestinal Inflammation in IBD. Inflamm Bowel Dis 21, 1297-1305 (2015).

9. Nakanishi Y, Sato T, Ohteki T. Commensal Gram-positive bacteria initiates colitis by inducing monocyte/macrophage mobilization. Mucosal immunology 8, 152-160 (2015).

10. Cohen BL, Sachar DB. Update on anti-tumor necrosis factor agents and other new drugs for inflammatory bowel disease. BMJ 357, j2505 (2017).

11. Barth MW, Hendrzak JA, Melnicoff MJ, Morahan PS. Review of the macrophage disappearance reaction. J Leukoc Bio/ 57, 361-367 (1995).

12. Zhang $\mathrm{N}$, et al. Expression of factor $\mathrm{V}$ by resident macrophages boosts host defense in the peritoneal cavity. J Exp Med 216, 1291-1300 (2019).

13. Smillie CS, et al. Intra- and Inter-cellular Rewiring of the Human Colon during Ulcerative Colitis. Cell 178, 714-730 e722 (2019). 
14. Mitsialis V, et al. Single-Cell Analyses of Colon and Blood Reveal Distinct Immune Cell

Signatures of Ulcerative Colitis and Crohn's Disease. Gastroenterology 159, 591-608 e510 (2020).

15. Boland BS, et al. Heterogeneity and clonal relationships of adaptive immune cells in ulcerative colitis revealed by single-cell analyses. Sci Immunol 5, (2020).

16. Chapuy L, et al. Two distinct colonic CD14(+) subsets characterized by single-cell RNA profiling in Crohn's disease. Mucosal immunology 12, 703-719 (2019).

17. Corridoni D, et al. Single-cell atlas of colonic CD8(+) T cells in ulcerative colitis. Nat Med 26, 1480-1490 (2020).

18. Sheng J, et al. Topological analysis of hepatocellular carcinoma tumour microenvironment based on imaging mass cytometry reveals cellular neighbourhood regulated reversely by macrophages with different ontogeny. Gut, (2021).

19. Jackson HW, et al. The single-cell pathology landscape of breast cancer. Nature $578,615-620$ (2020).

20. Guo N, et al. A 34-Marker Panel for Imaging Mass Cytometric Analysis of Human Snap-Frozen Tissue. Front Immunol 11, 1466 (2020).

21. Ijsselsteijn ME, van der Breggen R, Farina Sarasqueta A, Koning F, de Miranda N. A 40-Marker Panel for High Dimensional Characterization of Cancer Immune Microenvironments by Imaging Mass Cytometry. Front Immunol 10, 2534 (2019).

22. Sheng J, et al. Topological analysis of hepatocellular carcinoma tumour microenvironment based on imaging mass cytometry reveals cellular neighbourhood regulated reversely by macrophages with different ontogeny. Gut, gutjnl-2021-324339 (2021). 
23. Schapiro D, et al. histoCAT: analysis of cell phenotypes and interactions in multiplex image cytometry data. Nat Methods 14, 873-876 (2017).

24. Muzaki AR, et al. Intestinal CD103CD11b dendritic cells restrain colitis via IFN-gamma-induced anti-inflammatory response in epithelial cells. Mucosal Immunol, (2015).

25. D'Antoni ML, et al. Effects of decorin and biglycan on human airway smooth muscle cell proliferation and apoptosis. Am J Physiol Lung Cell Mol Physiol 294, L764-771 (2008).

26. von Moltke J, Ji M, Liang HE, Locksley RM. Tuft-cell-derived IL-25 regulates an intestinal ILC2epithelial response circuit. Nature 529, 221-225 (2016).

27. Krzyzak L, et al. CD83 Modulates B Cell Activation and Germinal Center Responses. Journal of immunology 196, 3581-3594 (2016).

28. Tumanov A, et al. Distinct role of surface lymphotoxin expressed by B cells in the organization of secondary lymphoid tissues. Immunity 17, 239-250 (2002).

29. Van Acker H, Coenye T. The Role of Reactive Oxygen Species in Antibiotic-Mediated Killing of Bacteria. Trends Microbio/ 25, 456-466 (2017).

30. Tarique AA, Logan J, Thomas E, Holt PG, Sly PD, Fantino E. Phenotypic, functional, and plasticity features of classical and alternatively activated human macrophages. Am J Respir Cell Mol Biol 53, 676688 (2015).

31. Yamada $\mathrm{H}$, et al. LPS-induced ROS generation and changes in glutathione level and their relation to the maturation of human monocyte-derived dendritic cells. Life Sci 78, 926-933 (2006). 
32. Ge J, et al. ROS-mediated apoptosis of HAPI microglia through p53 signaling following PFOS exposure. Environ Toxicol Pharmacol 46, 9-16 (2016).

33. Sawyer RT, et al. Beryllium-stimulated reactive oxygen species and macrophage apoptosis. Free Radic Biol Med 38, 928-937 (2005).

34. Baumgart DC, Carding SR. Inflammatory bowel disease: cause and immunobiology. Lancet 369, 1627-1640 (2007).

35. Schurch CM, et al. Coordinated Cellular Neighborhoods Orchestrate Antitumoral Immunity at the Colorectal Cancer Invasive Front. Cell 183, 838 (2020).

36. Browaeys R, Saelens W, Saeys Y. NicheNet: modeling intercellular communication by linking ligands to target genes. Nat Methods 17, 159-162 (2020).

37. Chapuy L, Sarfati M. Single-Cell Protein and RNA Expression Analysis of Mononuclear Phagocytes in Intestinal Mucosa and Mesenteric Lymph Nodes of Ulcerative Colitis and Crohn's Disease Patients. Cells 9, (2020).

\section{Figures}



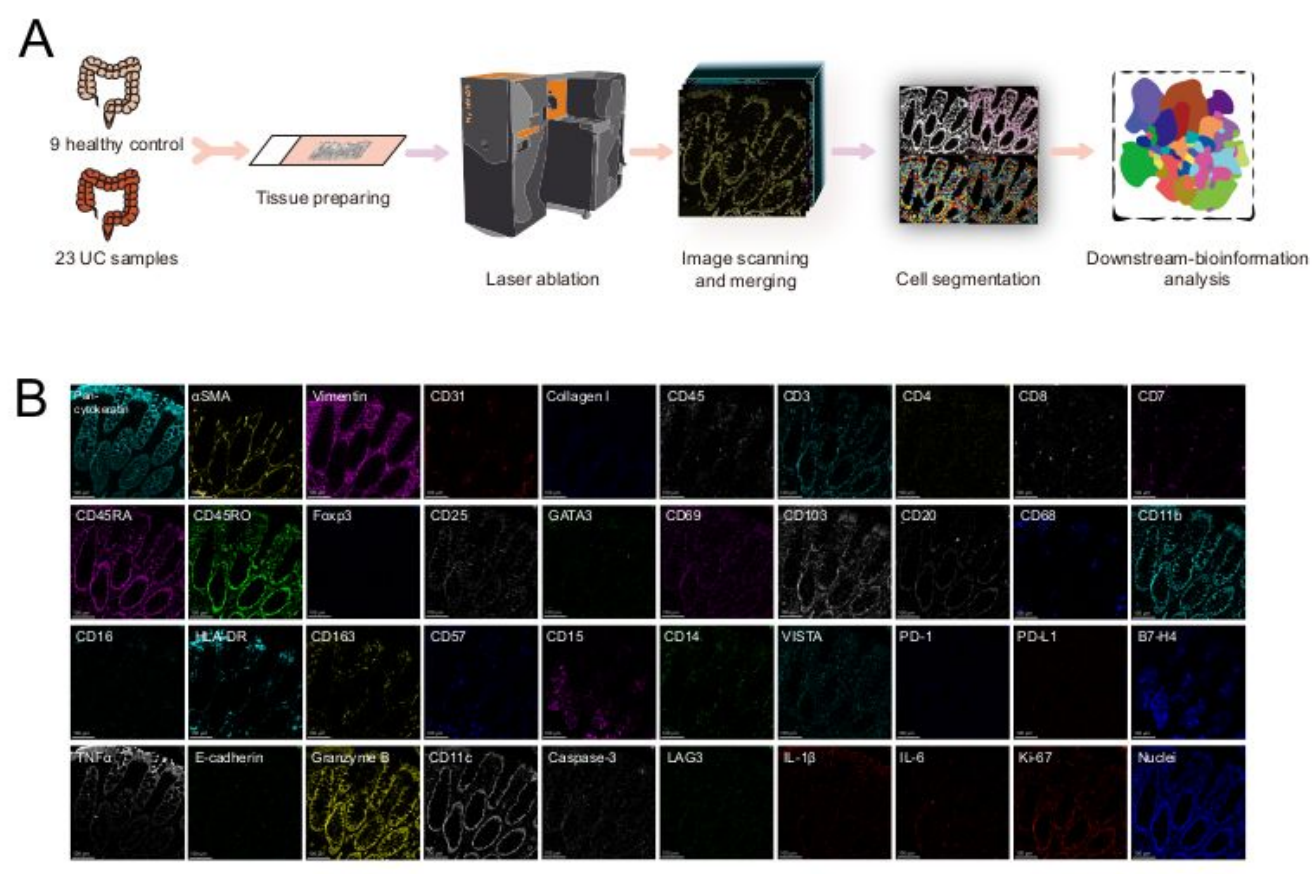

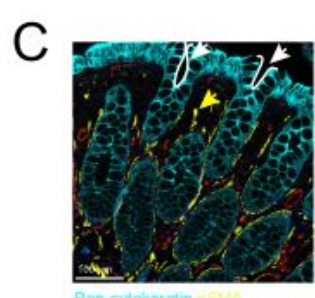

Collagen I CD31

$\mathrm{F}$
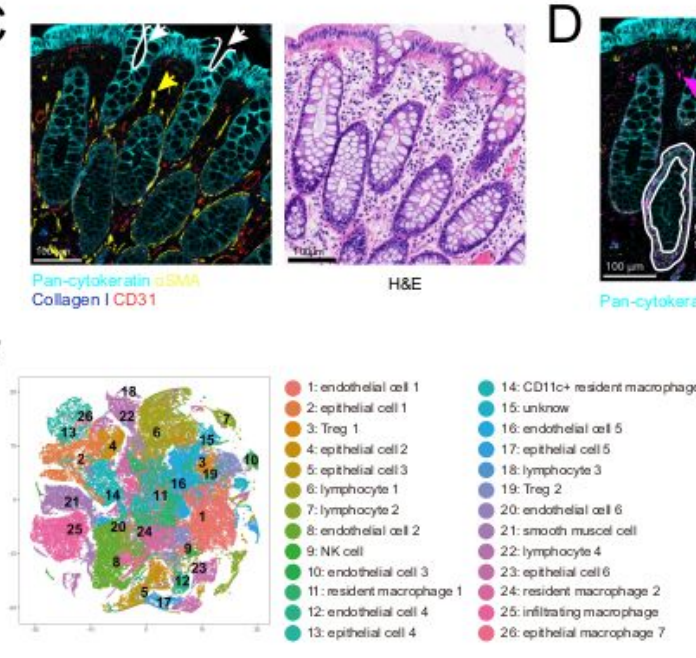

H\&E

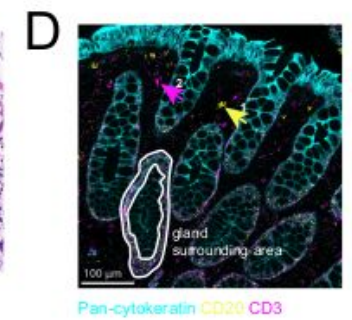

G

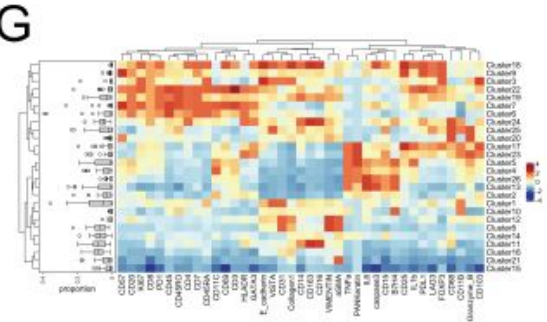

\section{Figure 1}

Representative mass cytometry images from a healthy donor sample using the 39-marker panel. A, The workflow of IMC. Patients' UC and self-control samples and healthy donor's samples were analyzed by IMC. B, Single color staining of the indicated marker above each plot. C, Pan-cytokeratin (cyan), aSMA (yellow), collagen I (blue), and CD31 (red) were used to portray the structure of colonic tissue (left image). H\&E staining (right image). Scale bars $100 \mu \mathrm{m}$. D, Pan-cytokeratin (cyan), CD20 (yellow), and CD3 
(magenta) highlight the distribution of T cells and B cells in colonic tissue. Arrow 1 (magenta) and arrow 2 (yellow) indicate the T cells and B cells, respectively. Scale bars $100 \mu \mathrm{m}$. E, CD68 (red), CD163 (blue) and CD11b (green) distinguish resident macrophages and inflammatory macrophages. Due to the overlap of colors, we used arrow 3 (plum) to highlight the resident macrophages (CD68+CD163+CD11b-) and arrow 4 (orange) to point out the inflammatory macrophages (CD68+CD163-CD11b+). Scale bars $100 \mu \mathrm{m}$. F, t-SNE plots were based on the single-cell data extracted from IMC images. 26 clusters of cells in ulcerative colitis samples were defined according their markers. G, The heat map showing the $z$-scored mean marker expression of 26 clusters with their frequency distribution pattern across different patients shown in box plot. 
A

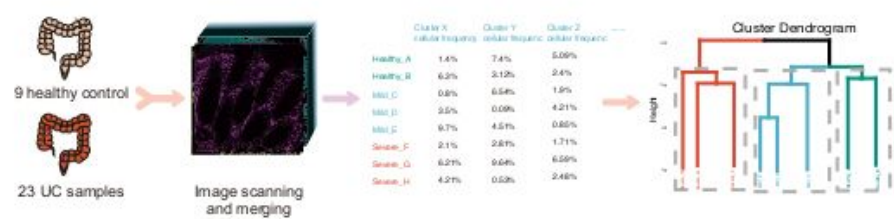

B
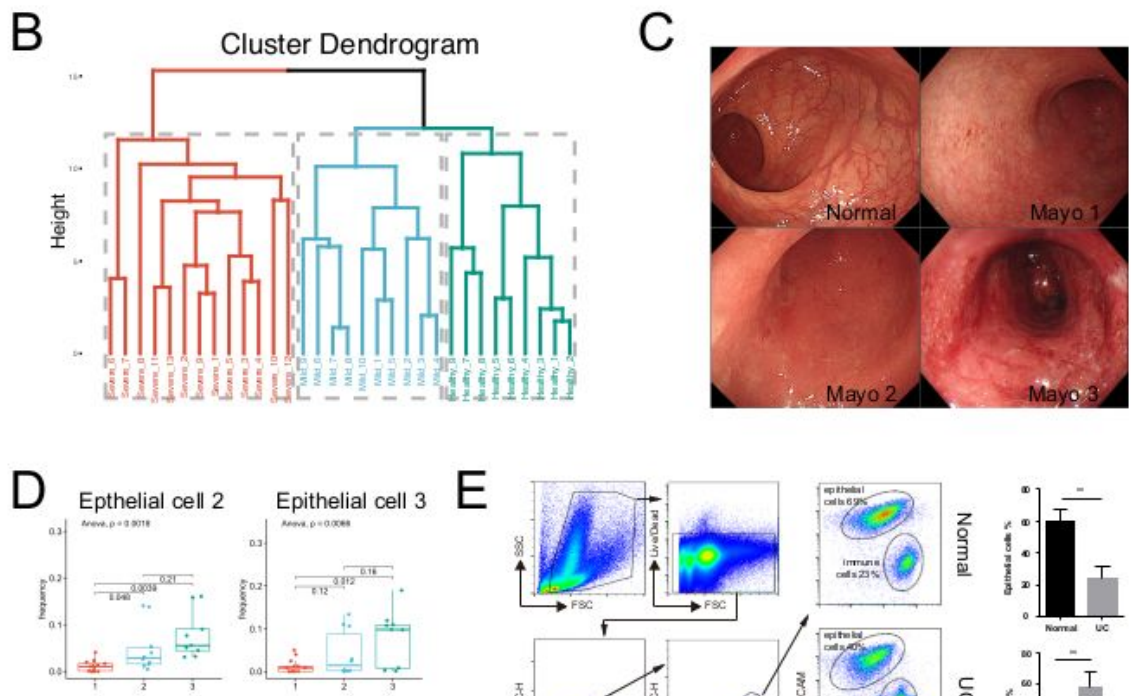

$\mathrm{E}$
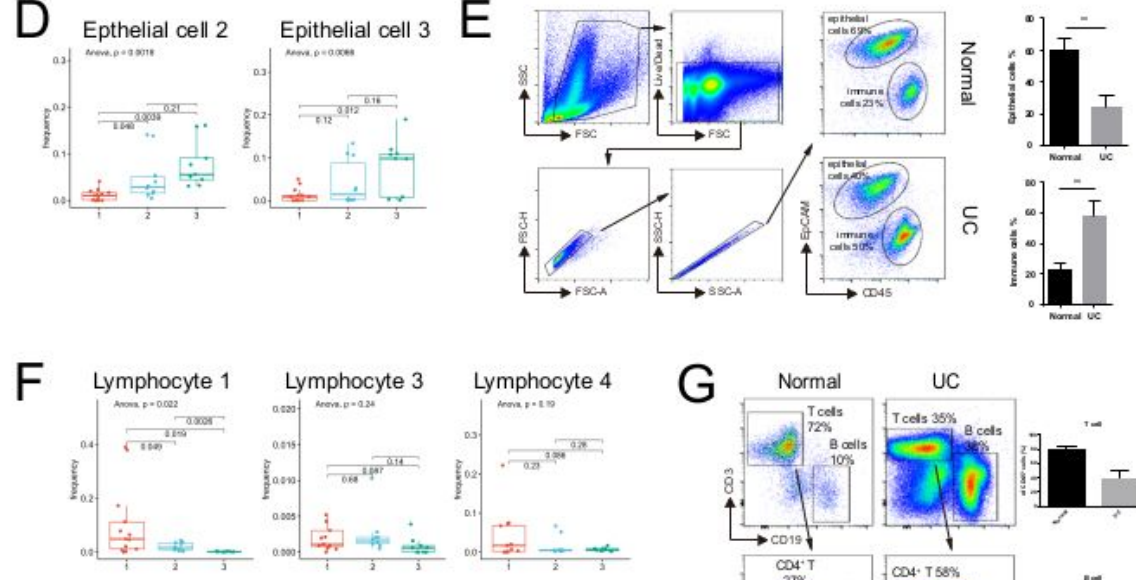

$\mathrm{H}$
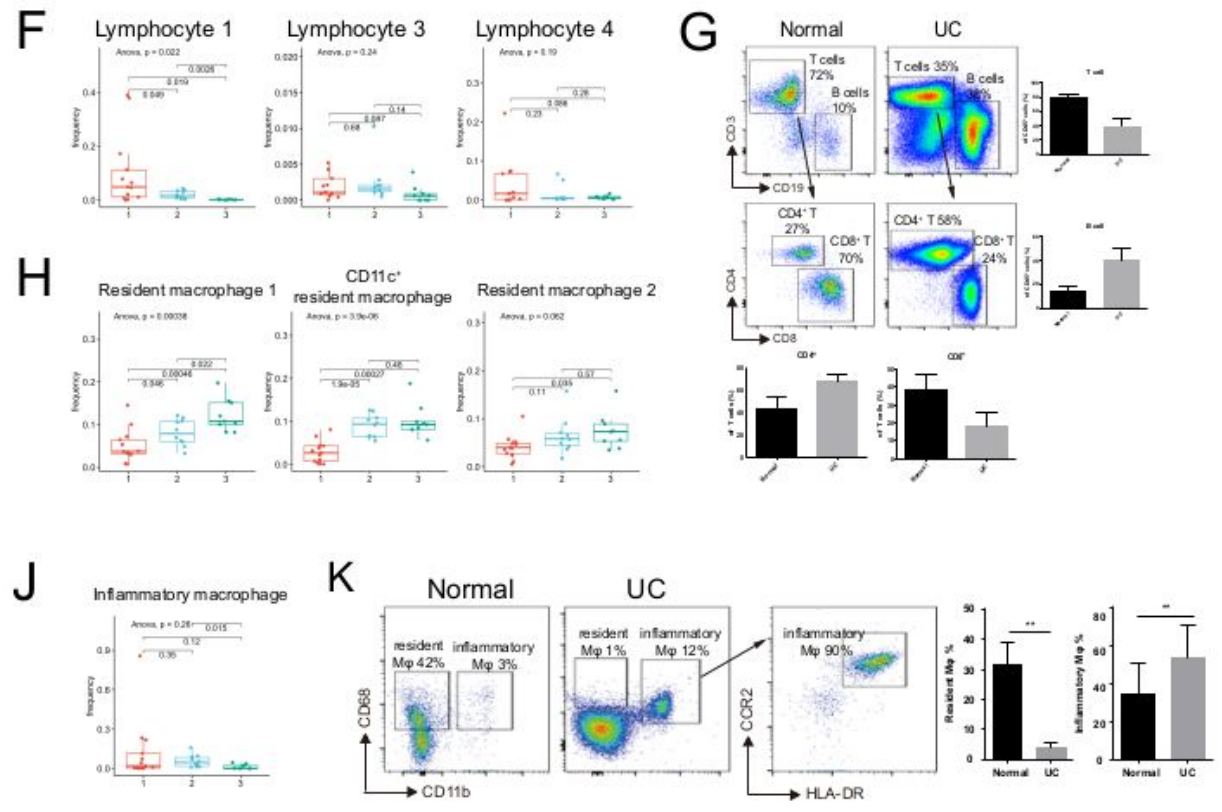

\section{Figure 2}

Intestinal ecosystem changes during UC progression. A and B 32 patient and donors' samples were separated into 3 major classes: severe patients (red), mild patients (blue), healthy patients (green) based on their cluster frequency. C, The images of 3 classes of UC samples determined by Mayo Clinic Endoscopic Subscore and normal donors. D and E, The distribution of epithelial cell 2 and 3 based on IMC results in C. And verification by FACS analysis of live samples, as shown in E. Statistic results are 
shown in the right panel of E. F and G, The distribution of lymphocyte 1, 3 and 4 based on IMC results shown in $F$ and verification by FACS analysis of live samples, as shown in $G$. $H$, The distribution of resident macrophage $1, C D 11 C+$ resident macrophage 1 , and resident macrophage 2 based on IMC results showed in $\mathrm{H}$. J, The distribution of inflammatory macrophages based on IMC results showed J. And verification by FACS analysis of live samples, as shown in K. Statistic results are shown in the right panel of $\mathrm{K}$.
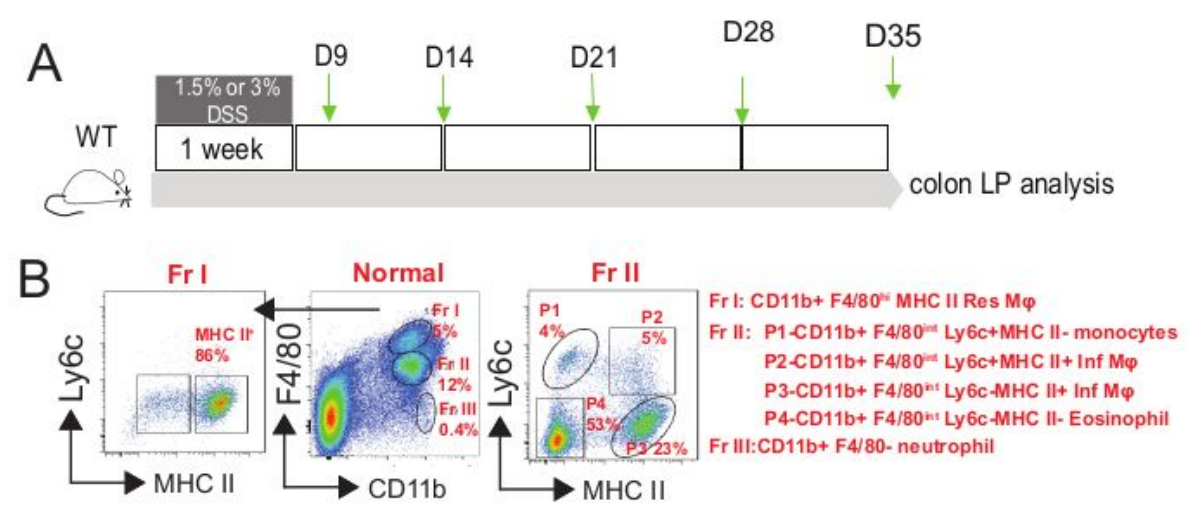

C

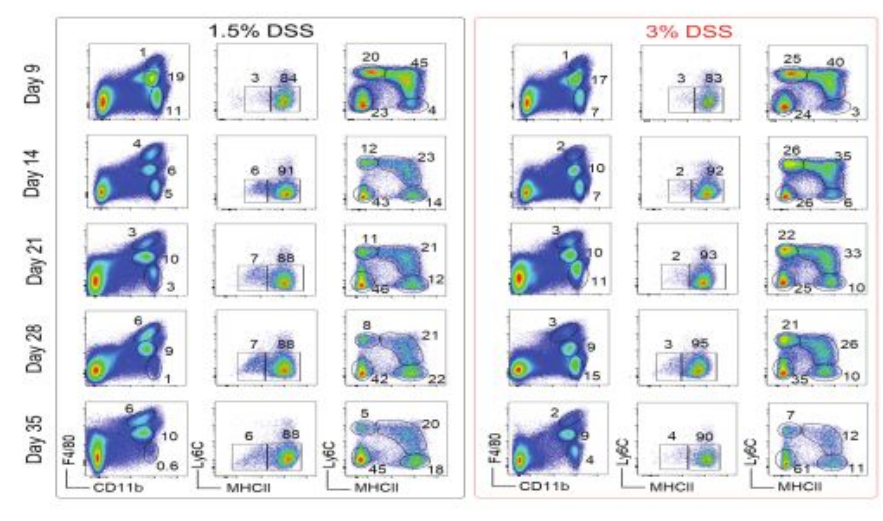

D
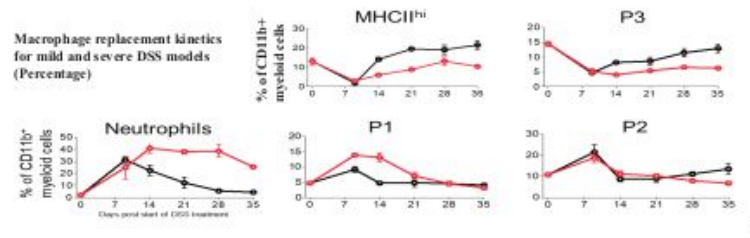

$E$
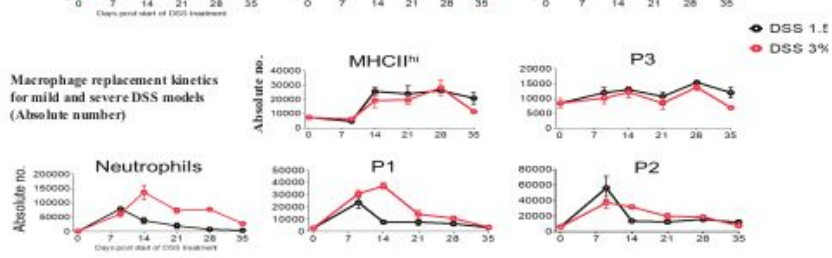

$\mathrm{F}$
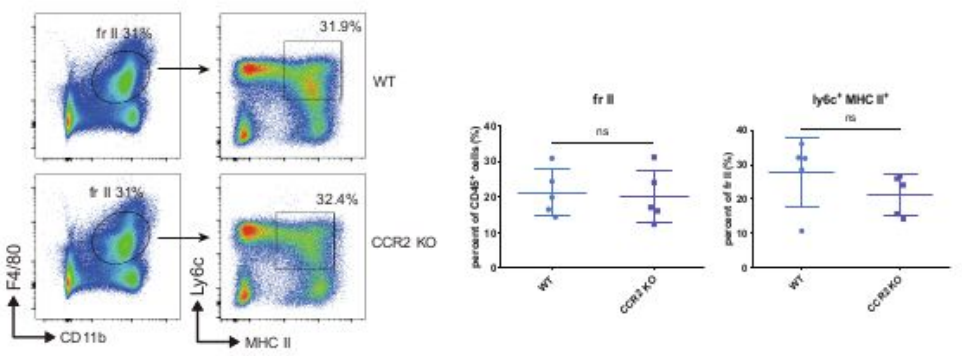

Figure 3 
Dynamics of myeloid cell changes during UC. A, DSS treatment schedule. Mice were treated with $1.5 \%$ or $3 \%$ DSS in drinking water, followed by a four-week chase period with normal drinking water. Colon tissue was isolated and analyzed at indicated time points. B, Gating strategy for different myeloid cells in normal colon tissue at day 0 . CD11b+ F4/80hi Fraction I cells were separated into $\mathrm{MHC} \mathrm{II-} \mathrm{and} \mathrm{MHC} \mathrm{II+}$ subsets of resident macrophages. CD11b+ F4/80int Fraction II cells were separated into P1 (Ly6c+ MHC II- monocytes), P2 (Ly6c+ MHC II+ inflammatory macrophage), P3 (Ly6c- MHC II+ infiltrating macrophage), and P4 (Ly6c- MHC II- eosinophil). CD11b+ F4/80- Fraction I cells were neutrophils. C, D and E, Representative FACS plots, subset percentages and absolute numbers for the different myeloid cells shown in Figure 5B during different time points of the DSS model. 
A

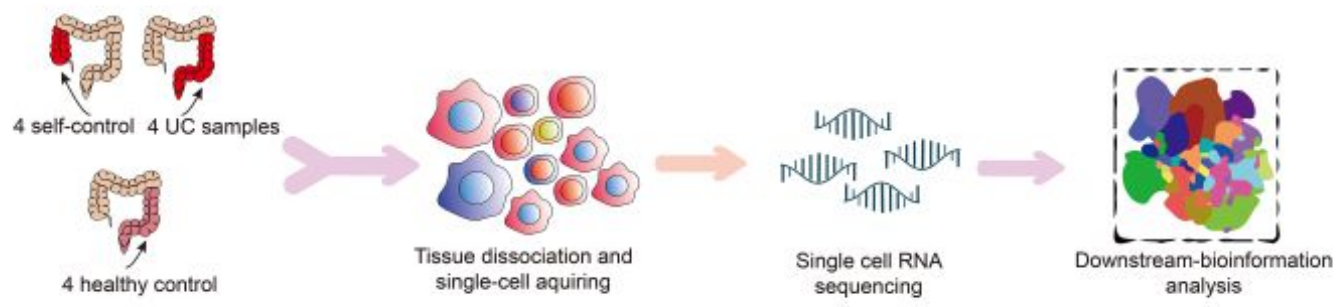

B
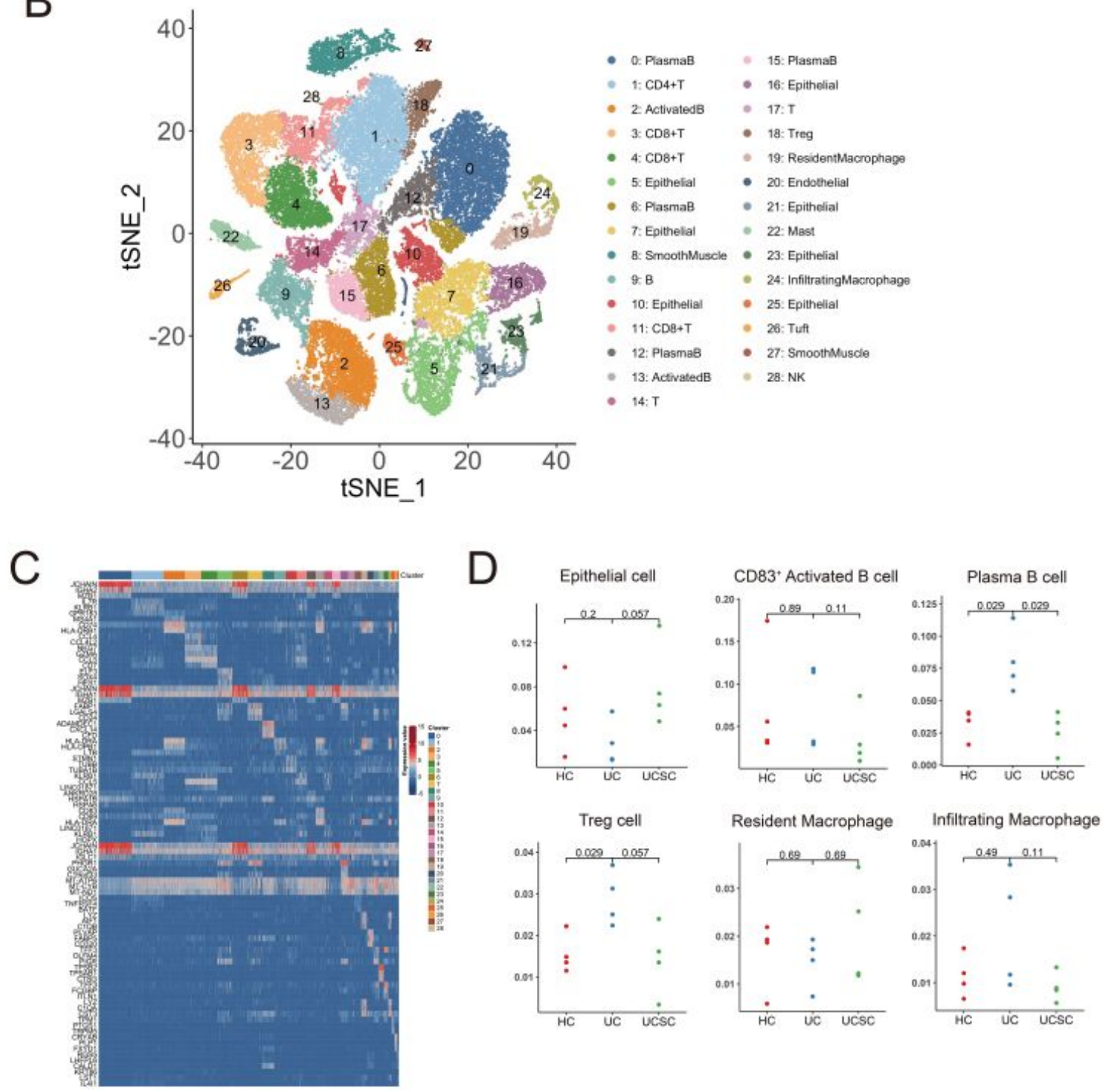

\section{Figure 4}

A single-cell transcriptomic and proteomic atlas of the immune microenvironment in UC. A, The workflow of scRNA-seq. Patients' UC and self-control samples and healthy donor's samples were analyzed by scRNA-seq. B, t-distributed stochastic neighbor embedding (t-SNE) plots based on the scRNA-seq data and 15 cell clusters of 12 samples were defined. C, The heat map showing the z-scored mean marker 
expression of 15 clusters. Color scale indicated normalized marker intensity. D, The charts reveals the distributions of epithelial cell and various immune cells among healthy donor, self-control and UC region.

A

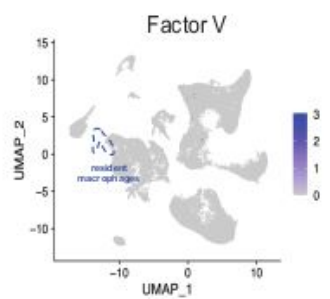

B

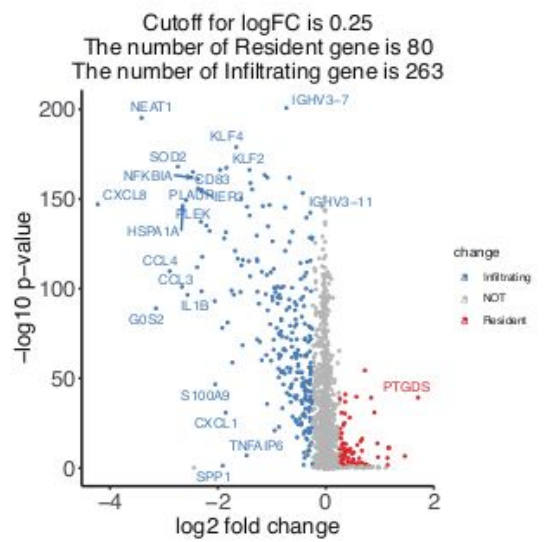

$E$

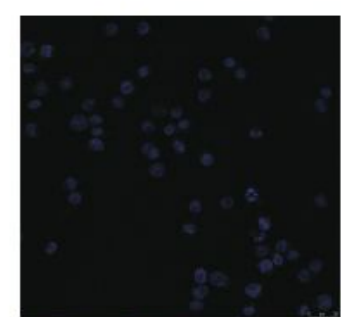

Nuclei ROS

C
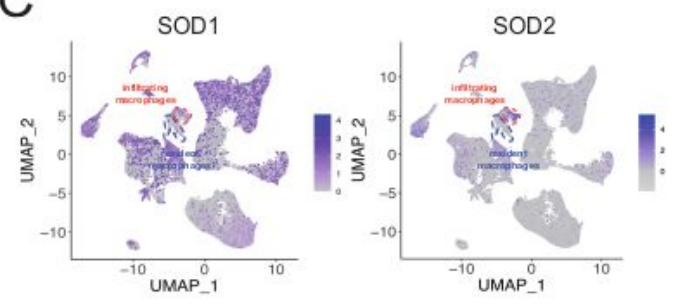

D

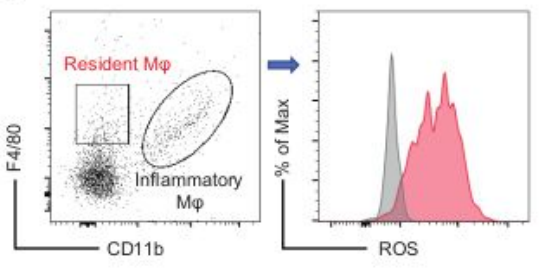

$\mathrm{F}$

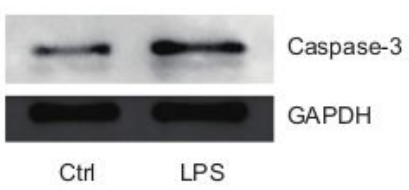

G
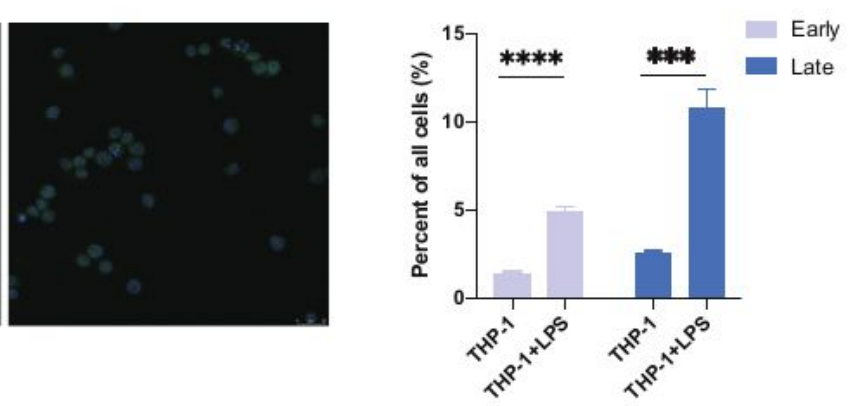

\section{Figure 5}

The mechanism of resident macrophage disappearance reaction A. The UMAP plot of Factor $\mathrm{V}$ expression based on scRNA-seq data. B. The differentially expressed gene (DEG) between resident macrophage and infiltrating macrophage. C. The UMAP plot of SOD1 and SOD2 expression in 
macrophages revealed by scRNA-seq analysis. Resident macrophage and inflammatory macrophage were highlighted with blue and red dot line. D. The different ROS production between two kinds of macrophages was measured using FACS compatible ROS probes based on UC patient sample. E, F and G. THP-1 cells were first induced to M0 state and then cocultured with LPS. ROS level was measured by immunofluorescence compatible probes in E. Activation of Caspase-3 was checked by western blot in F. Apoptosis was analyzed by Annexin V/PI staining and statistical results were shown in G.
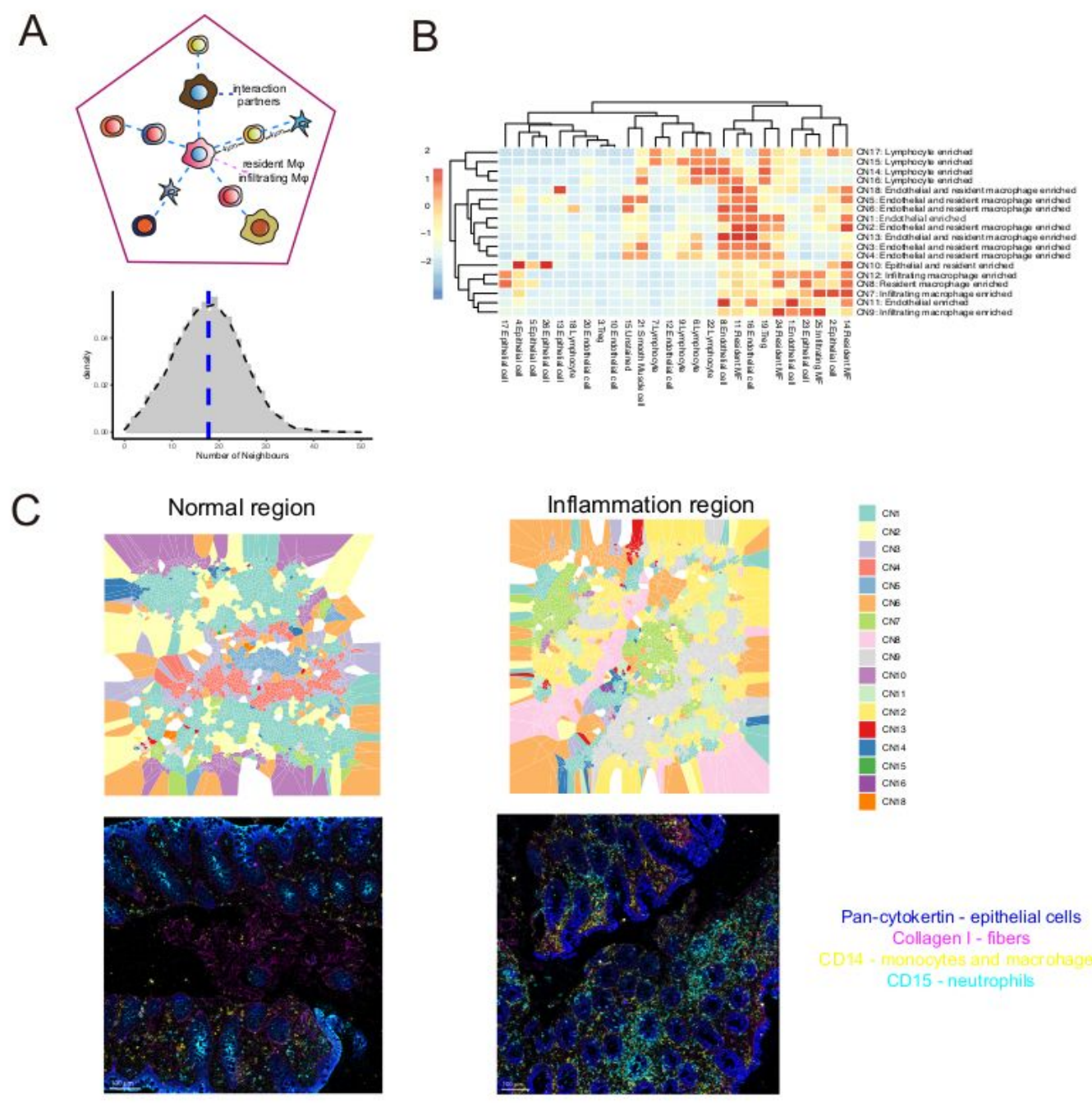

Pan-cytokertin - epithelial cells Collagen I - fibers

CD15 - neutrophils

D

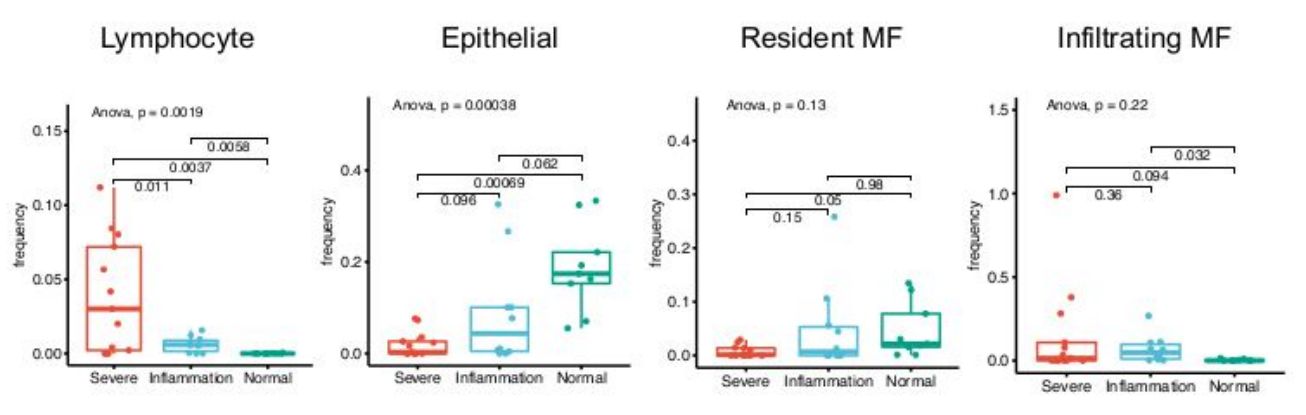


Cellular neighborhood changes during UC. A, Interaction partner detection model used to identify neighborhood cells within $4 \mu \mathrm{m}$ of the central cell. B. Eighteen major types of $\mathrm{CN}$ were annotated by the major cell type within $\mathrm{CN}$ and clustered by the scaled frequency of each type in $\mathrm{CN}$. C. The topology of IMC image was represented by Voronoi plot with various $\mathrm{CN}$ indicated by different colour. Representative IMC and Voronoi plot for normal region and inflammation region were shown. D. Comparison of lymphocyte-enriched $\mathrm{CN}$, infiltrating macrophage centered $\mathrm{CN}$, epithelial-enriched $\mathrm{CN}$, and resident MFenriched $\mathrm{CN}$ among different tissues.

A

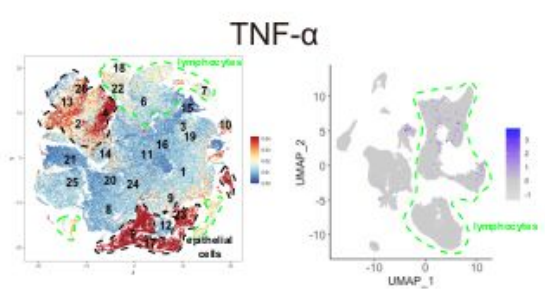

C

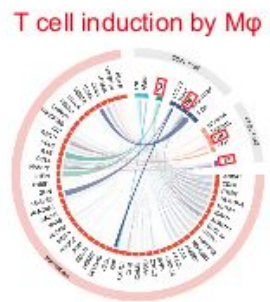

$E$

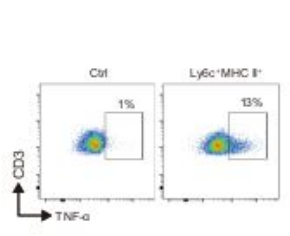

B cell induction by $M \varphi$

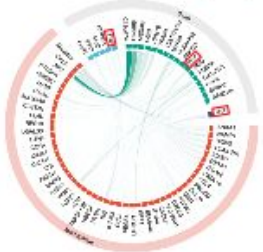

F

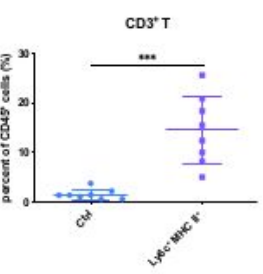

B

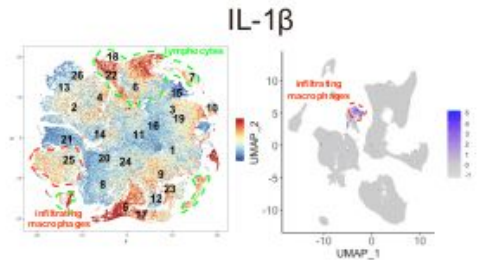

D

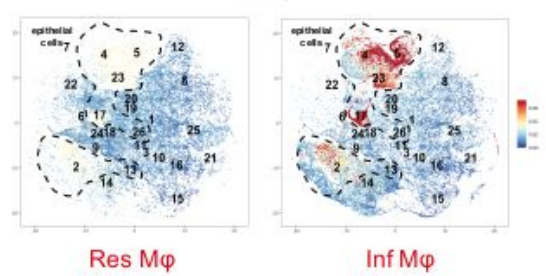

$\operatorname{Res} M \varphi$
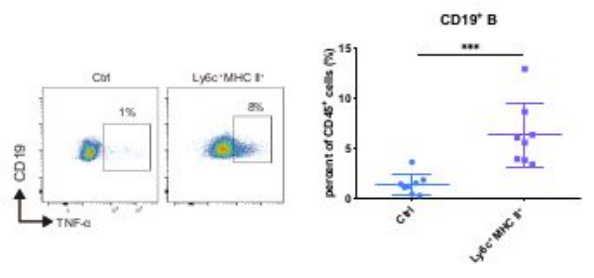

TNF-a
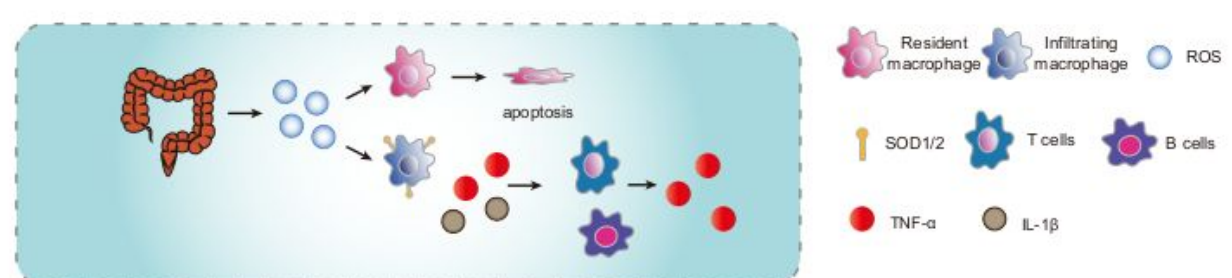


\section{Figure 7}

Infiltrating macrophages recruitment forms the inflammatory cytokine network within the cellular neighborhood. A, TNF-a expression pattern shown by t-SNE plots based on IMC (left panel) and scRNAseq (right panel) analysis. Epithelial cells are highlighted with black dotted lines, lymphocytes with green dotted lines, resident macrophages with blue dotted lines and inflammatory macrophages with red dotted lines. B, IL-1 $\beta$ expression pattern shown by t-SNE plot based on IMC (left panel) and scRNA-seq (right panel) analysis. C, Circos plot showing the cell-cell interactions between inflammatory macrophages and T cells (left panel) and inflammatory macrophages and B cells (right panel). Cell-cell interactions were performed by NicheNET. Macrophages (sender, outer ring)'s ligands engaged with receptors on T cells and $B$ cells (receiver, outer ring) and induced target genes (inner ring). Different colors of $T$ cells and $B$ cells indicate different subsets within T cells and B cells. D, TNF-a expression pattern of the interaction partners of resident macrophages (the left panel) and inflammatory macrophages (the right panel). $\mathrm{E}$, Ly6c+ MHC II+ inflammatory macrophages were sorted from colon in DSS treated mouse and cultured with lymphocytes from MLN. Representative plots and statistics results are shown for TNF-a production from $C D 3+T$ cells and $C D 19+B$ cells after the co-cultivation. $F$, The abstract diagram interprets the mechanism of resident macrophage disappearance effect. ROS was elevated as the first defense of the invading bacteria. At the same time, immune suppressive resident macrophage was wipe out purposely due to their sensitivity to ROS and replaced by inflammatory macrophage which was resistant to ROS based on high level of SOD1/2. Furthermore, inflammatory macrophages played a key role in forming the inflammatory cellular network by producing TNF- $\alpha$ and IL-1 $\beta$.

\section{Supplementary Files}

This is a list of supplementary files associated with this preprint. Click to download.

- SupplementaryFigureS2.pdf

- SupplementaryTableS2.xlsx

- SupplementaryTableS1.xlsx

- SupplementaryFigureS1.pdf 